\title{
Volt/VAR Optimization Function with Load Uncertainty for Planning of MV Distribution Networks
}

\author{
S. Rahimi ${ }^{1,2}$ \\ (1) ABB Enterprise Software \\ Västerås, Sweden \\ saeed.rahimi@se.abb.com
}

\author{
S. Massucco $^{2}$, F. Silvestro ${ }^{2}$ \\ (2) UNIGE, University of Genova \\ Genova, Italy
}

\author{
M.R. Hesamzadeh ${ }^{3}$, Y. Tohidi ${ }^{3}$ \\ (3) KTH, Royal Institute of Technology \\ Stockholm, Sweden
}

\begin{abstract}
Volt/VAR Optimization (VVO) function is an important element in real time operation of distribution networks and major part of advanced Distribution Management Systems (DMS). From planning prospective, VVO function can be used to optimize reactive power flow in distribution network to recommend the best operating condition for the control equipment in a predefined period of time in future (i.e. 24 hour). The typical objective function of VVO functions are minimizing the total system loss for a certain system load level. VVO function computes the optimized setting for transformer on-load tap changers (OLTC), Voltage Regulators (VR), and Capacitor Banks, while system voltage profile is maintained within its limits. In this paper the objective is to develop a planning VVO engine which can calculate the most probable expected loss of the network for the next 24 hours, and can recommend the best expected operating condition for the control equipment. For the VVO algorithm a full mixed integer linear programming (MILP) model is used to solve the loss objective of VVO problem for a planning application. The load uncertainty is modeled by an ARMA model which can create any arbitrary number of forecasted load scenarios to be used by VVO engine (implemented in a commercial solver GAMS, "General Algebraic Modeling System"). The implemented models have been tested on a real distribution network in southern Sweden and the results are presented.
\end{abstract}

Index Terms-- Distribution systems, Mixed Integer Linear Programming (MILP), Volt/VAR Optimization (VVO).

\section{INTRODUCTION}

Advanced Distribution Management System (DMS) applications are critical elements for operating the distribution system within "smart grid" vision [1]. In active distribution systems the operating condition requirements are different from the past where the new challenges are the large share of renewable energy resources connected to Medium Voltage (MV) level, or load scenarios due to Electric vehicles (EV) or other active loads. In order to optimize the total system loss, as one of Smart Grid major drives, the focus will be on reducing the total system losses and improve the grid efficiency. So to minimize the reactive power flow it is necessary to compensate the reactive power locally by means of compensation equipment such as capacitor banks. While it is also necessary to keep the voltage within an acceptable range. In operating distribution system, this task is called Volt/VAR Optimization (VVO) function [2].

VVO function is designed to optimize the voltage and reactive power flow in distribution network by using possible control equipment such as transformer on-load tap changers (OLTC), Voltage Regulators (VR), and switching the Capacitor Banks [3],[4]. As a general description the VVO problem is a mixed-integer nonlinear deterministic optimization problem with large number of variables and constraints [5][6][7]. Looking at the literature, it is possible to find the wide range of methods and algorithms, as we have discussed in our recent works [8][9].

The VVO function is used both for real-time operation of DNs and also operation planning where the objective is to recommend the best practical operating condition for the control equipment while minimizing the total loss. As continuation to our previous practice [10], in this paper we use the mixed integer linear programming (MILP) for modeling the VVO problem and have extended it for next 24 hour (day ahead planning). The original attempt for modeling VVO problem by MILP was taken from recent research paper [11].

The main contribution of this paper is developing a decoupled stochastic planning VVO engine which can calculate the most probable expected loss of the network for the next 24 hours, and can recommend the best expected operating condition for the control equipment. For modeling the load uncertainty and creating load scenarios and ARMA modeling is deployed. The full MILP planning VVO engine is implemented in a commercial solver GAMS, "General Algebraic Modeling System [12]". The Output will be the most probable expected setting for regulating equipment which yields into minimum loss over a period of time (i.e 24 hours). The implemented models have been tested on a real distribution network in southern Sweden. This paper is outlined as following: in section 2 the theoretical background for MILP modeling of Volt/VAR optimization for planning problems are given. In section 3 our proposed method for stochastic VVO for planning applications and including the load uncertainty is explained. In section 4 the detail of the real distribution test system under study is provided and results from a planning VVO with load uncertainty modeling is presented. 


\section{THE MILP MODEL FOR PLANNING VVO}

In this section the theoretical background of a planning Volt/VAR optimization problem by a full MILP technique is given. The distribution network is assumed to have $\mathrm{N}+1$ nodes (or buses) including one slack bus which we assign it to connected bus to the substation. In the network there are $N_{b}$ branches (lines or transformers). Voltages and currents are represented by the Cartesian coordinates of their phasors $\left(V^{\text {re }}\right.$, $\left.V^{\mathrm{im}}\right)$ and $\left(I^{\mathrm{re}}, I^{\mathrm{im}}\right)$, respectively. As usually we assume that voltage phasor at the slack bus is a reference, so $V s$ is equal to rated value and phase angel equal to zero. All the equations are written in per unit. The Objective function can be formulated as:

$\sum_{h r=1}^{24}\left(\sum_{b \in B_{s}} V_{s} I_{b, h r}^{r e}+w_{\min } \sum_{k=1}^{N} \chi_{k, h r}^{\min }+w_{\max } \sum_{k=1}^{N} \chi_{k, h r}^{\max }+\sum_{k \in K_{\mathrm{L}}} w_{k}^{\mathrm{L}} u_{k, h r}^{\mathrm{L}}+\sum_{k \in K_{\mathrm{EG}}} w_{k}^{\mathrm{EG}} u_{k, h r}^{\mathrm{EG}}\right)$

where $B_{s}$ is the set of all branches (feeders) connected to the substation slack bus, $h r$ is the set of hours for next day, $I_{b}$ indicates the current in branch $b$; and $\chi_{k, h r}^{\min }$ and $\chi_{k, h r}^{\max }$ are the square of the violations of the minimum and maximum voltage limits at node $k$ for each hour $h r$, respectively. In order to assign penalty to voltage violation we define $w_{\min }$ and $w_{\max }$ are the weights to the corresponding violations; $K_{\mathrm{L}}$ and $K_{\mathrm{EG}}$ are the set of nodes representing the connection point of any load or embedded generation (EG).

\section{A. Power flow equations for Branches}

In order to calculate the power flow in the network, we need to write the bus and branch equations separately, while we include the linearized equations for all the equipment in the network. Starting with branch equation, we assume that the branch $b$ is a line while the sending node is $h$ and receiving node is $k$. Three current phasors can be written for this branch as: $I_{b}$ for the current flow through the impedance of the branch (assuming one-phase PI equivalent circuit), $I_{b, h}^{\text {sh }}$ and $I_{b, k}^{\text {sh }}$ for currents flow into the shunt admittance connected to sending node $h$ and receiving node $k$, respectively.

For the case that the branch $b$ is a line, parameters of the one-phase PI equivalent circuit are: resistance $R_{b}$, reactance $X_{b}$ and two equal susceptances $B_{b}$ that for the line shunt capacitances $C_{b}$. For the cases that branch $b$ is a transformer, parameters are $R_{b}$ and $X_{b}$ for the resistance and reactance of the transformer windings (referred to the primary side) and $B_{b}$ is the magnetizing shunt susceptance.

By equation 2, we can calculate the real and imaginary parts of current $I_{b, h, h r}^{\text {sh }}$ of each branch $b$ at bus $h$ :

$I_{b, h, h r}^{\mathrm{sh}, r e}+B_{b} V_{h, h r}^{i m}=0 \quad$ and $\quad I_{b, h, h r}^{\mathrm{sh}, i m}-B_{b} V_{h, h r}^{r e}=0$

By equations (3), we can calculate voltage phasors difference at the terminals $h$ and $k$, when the branch $\mathrm{b}$ is a line (or a fixed- ratio transformer):

$$
\begin{aligned}
& V_{h, h r}^{r e}-V_{k, h r}^{r e}-R_{b} I_{b, h r}^{r e}+X_{b} I_{b, h r}^{i m}=0 \\
& V_{h, h r}^{i m}-V_{k, h r}^{i m}-X_{b} I_{b, h r}^{r e}-R_{b} I_{b, h r}^{i m}=0
\end{aligned}
$$

By equations (4), we can calculate the constraint on the voltages at tap position $t$, for LTC transformers:

$$
\begin{aligned}
& V_{h, h r}^{r e}-r_{b, t} \cdot V_{k, h r}^{r e}-R_{b} I_{b, t, h r}^{r e}+X_{b} I_{b, t, h r}^{i m}+\Delta v_{b, t, h r}^{r e}=0 \\
& V_{h, h r}^{i m}-r_{b, t} \cdot V_{k, h r}^{i m}-X_{b} I_{b, t, h r}^{r e}-R_{b} I_{b, t, h r}^{i m}+\Delta v_{b, t, h r}^{i m}=0
\end{aligned}
$$

In equation (4), the $I_{b, t, h r}^{r e}$ and $I_{b, t, h r}^{i m}$ are the hourly phasor of current at the primary side $h$ for the tap position $t$ of transformer (with corresponding turn ratio $r_{b, t}$ ). We define a binary variable $u_{b, t, h r}$ for each tap position $t$ in hour $h r$, in a way that $t$ is the selected tap position, then the binary variable $u_{b, t}$ will be equal to 1 , and it will be zero for all other tap positions.

For the tap changers, another set of binary variable are needed. Assuming $t$ is the selected tap position $\left(u_{b, t, h r}=1\right)$, we need to define two auxiliary variables $\Delta v_{b, t, h r}^{r e}$ and $\Delta v_{b, t, h r}^{i m}$ (for each hour) equal to zero when their value should adapt to transformer terminals voltage difference if $u_{b, t, h r}=0$. In that case if $I_{b, t, h r}^{r e}$ and $I_{b, t, h r}^{i m}$ are forced to be zero for $u_{b, t, h r}=0$, we can calculate $I_{b, h r}$ as the summation of all $I_{b, t, h r}$. Assuming $n_{b, \mathrm{t}}$ is the number of tap positions and $I_{b}^{\max }$ is the maximum feasible value of $\left|I_{b}\right|$, the set of constraints is as following:

$$
\begin{gathered}
\Delta v_{b, t, h r}^{r e}+B N u_{b, t, h r} \leq B N \text { and }-\Delta v_{b, t, h r}^{r e}+B N u_{b, t, h r} \leq B N \\
\Delta v_{b, t, h r}^{i m}+B N u_{b, t, h r} \leq B N \text { and }-\Delta v_{b, t, h r}^{i m}+B N u_{b, t, h r} \leq B N \\
-I_{b}^{\max } u_{b, t, h r} \leq I_{b, t, h r}^{r e} \leq I_{b}^{\max } u_{b, t, h r} \text { and }-I_{b}^{\max } u_{b, t, h r} \leq I_{b, t, h r}^{m} \leq I_{b}^{\max } u_{b, t, h r}
\end{gathered}
$$

for all above constraints $\forall t=1 \ldots n_{b, t}$

$$
\sum_{t=1}^{n_{t}} u_{b, t, h r}=1, \quad I_{b, h r}^{r e}-\sum_{t=1}^{n_{b, t}} I_{b, t, h r}^{r e}=0 \quad \text { and } \quad I_{b, h r}^{i m}-\sum_{t=1}^{n_{b, t}} I_{b, t, h r}^{i m}=0
$$

where $\mathrm{BN}$ is an adequately large number.

\section{B. Power flow equations for Bus equations}

For each bus (node $k$ ) we can define three current variables $I_{\mathrm{L}, k, h r}, I_{\mathrm{C}, k, h r}, I_{\mathrm{EG}, k, h r}$ for each hour $h r$ that represents the currents flowing into connected load, capacitor bank and current injected by the generator to node $k$, respectively. It is assumed that connected bus to substation is slack bus $s$ with voltage as $V_{s}=\left|V_{s}\right| e^{j 0}$. Considering the typical sign convention for the current directions, the Kirchhoff law can be written as following:

$$
\begin{aligned}
& \sum_{b \in B_{k}^{+}} I_{b, h r}^{r e}-\sum_{b \in B_{k}^{-}} I_{b, h r}^{r e}+\sum_{b \in B_{k}} I_{b, k, h r}^{\mathrm{sh}, r e}+I_{\mathrm{L}, k, h r}^{r e}+I_{\mathrm{C}, k, h r}^{r e}-I_{\mathrm{EG}, k, h r}^{r e}=0 \\
& \sum_{b \in B_{k}^{+}} I_{b, h r}^{i m}-\sum_{b \in B_{k}^{-}} I_{b, h r}^{i m}+\sum_{b \in B_{k}} I_{b, k, h r}^{\mathrm{sh}, i m}+I_{\mathrm{L}, k, h r}^{i m}+I_{\mathrm{C}, k, h r}^{i m}-I_{\mathrm{EG}, k, h r}^{i m}=0
\end{aligned}
$$


In above equation $B_{k}$ is the set of lines $b$ connected to node $k$, where we distinguish between the set of lines leaving node $k$ as $B_{k}^{+}$and the set of lines entering in node $k$ as $B_{k}^{-}$. When the node $k$ is connected to the secondary side of a LTC transformer, $I_{b, h r}^{r e}$ and $I_{b, h r}^{i m}$ in the second summation are respectively equal to $\sum_{t=1}^{n_{t}} r_{b, t} I_{b, t, h r}^{r e}$ and $\sum_{t=1}^{n_{t}} r_{b, t} I_{b, t, h r}^{i m}$ for ecah hour $h r$.

\section{Linear Model for all equipment in the network}

Finally a similar linearization method is applied to model the different equipment in the network such as Load, embedded generators and shunt capacitors. The complete formulation of equipment models can be recalled in [9].

\section{Branch and node constraints}

In each branches of the network, the branch current is limited in such a way that to satisfy both the limit in the current capacity of the network that the maximum allowable current on the transformer windings. These limitations have been implemented in the model, by means of a set of linear constraints; each of them corresponds to a side of the regular polygon inscribed in the circle of radius equal to the maximum value of allowed current. By using a 23-sided polygon, the error due to this approximation is lower than $1 \%$. The complete set of branch/node constraints can be recalled in [11]. It is noted that when the optimization is run over a period of time, it is desirable to limit (minimize) the number of switching for capacitor bank status and also number of tap steps change [13]. Since the target in this paper is to calculate the expected setting for control equipment, here we have not limited the number of switching over 24 hours, though this can be a valid and practical constraint for the model.

\section{MODELING STOCHASTIC VVO FOR PLANNING APPLICATIONS}

\section{A. Load uncertainty model versus deterministic load}

In the previous section the MILP model for a planning VVO problem is based on a set of fixed load values for each separate hour. The assumption of a set of deterministic load values is a valid assumption since DSOs always rely on historical load values and use typical load profiles for many planning applications. Still to improve the model, it is interesting to expand the VVO problem by modeling some load uncertainty as an input. When the target is to minimize the loss over a period of time, it is desirable to utilize a realistic set of values for load over that period. As a result it is necessary to take advantage of historical load data and find proper load scenarios where load uncertainty is considered.

Our early effort showed that a cross-sectional method which produces large number of samples for load of each hour cannot be useful because of the nature of residential load and the correlation between the loads of consequent hours. So we have chosen a time series based stochastic model for the load. Among different methods for modeling the load as a time series an ARMA process (auto-regressive moving average) is chosen which is widely used approach for forecasting the load and considering the random component in it [14][15].

A typical ARMA model is denoted as $\operatorname{ARMA}(p, q)$ which are the order of model. The parameter identification for ARMA model can be done by a recursive scheme, or using a maximum-likelihood approach as we have used in this research. $\operatorname{ARMA}(p, q)$ is combination of AR (autoregressive of order " $p$ ") and MA (moving-average of order " $q$ ") is formulated as following [16]:

$$
\begin{aligned}
\operatorname{ARMA}(p, q): X_{t}= & \beta_{1} X_{t-1}+\beta_{2} X_{t-2}+\ldots+\beta_{p} X_{t-p}+ \\
& e_{t}+\alpha_{1} e_{t-1}+\alpha_{2} e_{t-2}+\ldots+\alpha_{q} e_{t-q}
\end{aligned}
$$

For creating load scenarios with the ARMA model, there are two main approaches for parameter identification of ARMA model: a recursive scheme, or using a maximumlikelihood approach, which is basically a non-linear regression algorithm. In this paper we have chosen to use the maximumlikelihood approach and the MATLAB solver is used for calculating both the order of the ARMA model and also the coefficients for the ARMA model using available historical load data.

Upper part of flow chart in figure 1 shows the steps for creating load scenarios with the ARMA model, as following:

Step 1: taking the hourly load profile of at least one complete year. In this work the historical data of year 2008 from the Gothenburg test system is used [17].

Step 2: identify the four different seasons and calculate four different seasonal "Standard Deviation" and "Probability distribution function (pdf)" from load profiles by using Akaike Information Criterion (AIC) [18][19] and maximum likelihood technique. For our test system, it is observed that one normal distribution can be fitted to the pdf histogram of each season. The characteristics of this normal distribution are used to calculate the parameters of white noise.

Step 3: By using the data from each season, and creating a loop to calculate the AIC matrix, the target is to calculate the best possible order for ARMA model which fits the data of each season. Here the AIC order is calculated by using the standard MATLAB solver (armax and aic).The results from the MATLAB solver show that 10x10 dimensions for the AIC matrix are accurate enough for modeling the data.

Step 4: Select one day in the historical data set from that season and the input above from the ARMA model to create a time series of 24 load values (load forecast) for that day of the season.

Step 5: Repeat the previous step any arbitrary number of time to create several diffident load scenario for that season. Here

\section{B. Stochastic Volt/VAR optimization}

In this paper a decoupled stochastic VVO function is proposed. It means that we analysis the load uncertainty outside the optimization process and the results from optimization of load scenarios are used to calculate the most probable system loss and expected setting for control 
equipment. Lower part of flow chart in figure 1 shows the steps of our proposed decoupled stochastic VVO method:

Step 1: taking the hourly load profile of at least one complete year to identify the 4 different seasons, use the maximum likelihood technique to calculate 4 different seasonal "Standard Deviation" and "Probability distribution function (pdf)" from the load profile. Histograms of the pdf for each season are used to calculate the probability of each time-series load scenario.

Step 2: based on the arbitrary number of load scenarios for each season, and by using the histogram for that season, it is possible to calculate the "Probability distribution function of each scenario", which is actually calculating the cumulative probability all load levels in that scenario and comparing the normalizing that summation to the probability of all scenarios:

$\pi_{s c_{i}}=\frac{\sum_{h r=1}^{24} \pi_{h r} L_{h r}^{s c_{i}}}{\sum_{s c=s c_{1}}^{s c_{n}}\left(\sum_{h r=1}^{24} \pi_{h r} L_{h r}^{s c_{i}}\right)}$

where $\pi_{s c_{i}}$ is probability of each scenario, $\pi_{h r}$ is the probability of load level in that season (taken from histogram), and $L_{h r}^{s c_{i}}$ is hourly load value.

Step 3: Feeding all of these load scenarios to VVO engine and calculating the total loss and optimized set-tings of control equipment (tap position, capacitor switch status, MVar level of EGs) for each of 24 hourly intervals of each scenario.

Step 4: Combining the calculated loss from each scenario and its probability to calculate the most probable (expected total loss) for next 24 hours for the system, as follows:

$E($ loss $)=\sum_{s c=s c_{1}}^{s c_{n}} \pi_{s c_{i}} \operatorname{Loss}_{s c_{i}}$

where $\operatorname{Loss}_{s c_{i}}$ is optimized loss value for each scenario.

Step 5: in a similar manner, combining the calculated control settings in the optimized solution of each scenario load (tap position, capacitor switch, MVar level of EG) from each scenario and its probability, to calculate the most probable control settings (expected control settings) for next 24 hours, as follows:

$$
\begin{aligned}
& E\left(u_{b, t, h r}\right)=\sum_{s c=s c_{1}}^{s c_{n}} \pi_{s c_{i}} \sum_{h r=1}^{24}\left(u_{b, t, h r}\right) \\
& E\left(u_{k, s w, h r}^{\mathrm{C}}\right)=\sum_{s c=s c_{1}}^{s c_{n}} \pi_{s c_{i}} \sum_{h r=1}^{24}\left(u_{k, s w, h r}^{\mathrm{C}}\right) \\
& E\left(u_{k, g, h r}^{\mathrm{EG}}\right)=\sum_{s c=s c_{1}}^{s c_{n}} \pi_{s c_{i}} \sum_{h r=1}^{24}\left(u_{k, g, h r}^{\mathrm{EG}}\right)
\end{aligned}
$$

where $u_{b, t, h r}, u_{k, s w, h r}^{\mathrm{C}}$ and $u_{k, g, h r}^{\mathrm{EG}}$ are calculated control settings for the optimized solution of tap positions, capacitor switching and Mvar level of EG respectively. After calculating the probability above for each tap step, each capacitor switch and Mvar level of EG, the figure occurring the most among them will be the most probable setting of the equipment. It is noted that in the summations above the probability of each scenario is always multiplied by zero for all settings other than the optimized one.

\section{SIMULATION AND DISCUSSIONS ON RESULTS}

\section{A. Test case: 30-Bus Gothenburg distribution Network}

Figure 2 shows the single line diagram of the test system used for this study which is a real distribution network located in the residential part of city of Gothenburg [17]. The network is $10 \mathrm{kV}$ with twenty-eight of $10 / 0.4-\mathrm{kV}$ substations, modeled as loads (consisting aggregated loads of small houses). The network is supplied from $130 \mathrm{kV}$ medium voltage substation and consists of three tie-switches which are normally open and split the network into 3 feeders. The MV level transformer is equipped with tap changer and we have modified the actual network with locating two capacitor banks and two small embedded generations on different feeders. All the line parameters are actual values from the $10 \mathrm{kV}$ cable network. In Table 1, the parameters of transformer, embedded generators and capacitors and peak load values are provided.

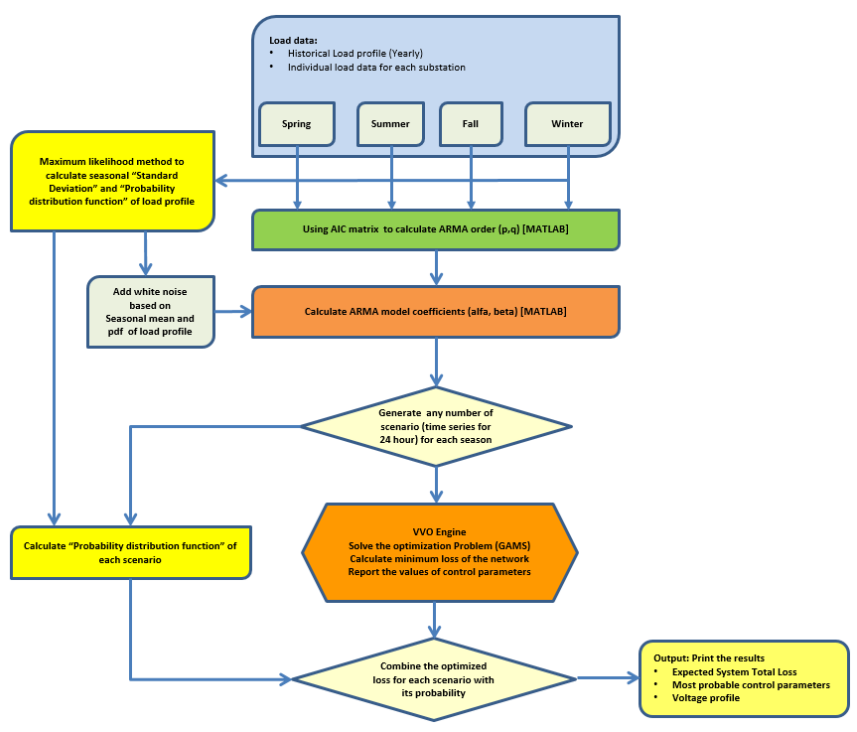

Figure 1. Flow chart of the proposed stochastic VVO method.

\section{B. Simulation and Results}

Table 2 is the result from stochastic VVO study applied for winter season and the details of simulation statistics. Figures 3 to 5 are the expected setting for control parameters within a typical day of winter season based on 20 scenarios under study. Figure 3 shows that for the typical day of winter, the highest tap position (t9) is the most probable position which yields in lowest loss. Other tap positions ( $t 7, \mathrm{t} 8)$ also have quite high probability and this facilitates the planning of operation, for example for the day-ahead planning problem. Similarly figure 4 shows that the desired capacitor switching status for the optimized solution (or the most probable status) is $s w 1$ for both capacitor banks. Referring to figure 5, the MVar levels provided by EG are mainly around $g 4$ setting which means that EG is not utilized for supplying MVar in the optimal solution. This could be due to small size of EG MVar 
supply to this network. The total expected loss from studying different load scenarios is around $7 \mathrm{MWh}$ for a typical winter day, according to table 2. Figure 6 shows the voltage profile for all three feeders for the most expected scenario (sc7). In fact there is no direct way to calculate the expected voltage profile from the stochastic optimization analysis unless the expected settings for regulating equipment are used for calculating the voltage profile. Here since the most probable scenario is scenario 7 , the voltage from that scenario is plotted. As can be seen the nodes on the second feeder have lower voltage relative to the other two feeders. This is useful information for planning purposes which indicates a voltage drop bottleneck in the network requiring remedial action such as the installation of capacitor banks or other compensators or a feeder re-configuration plan.

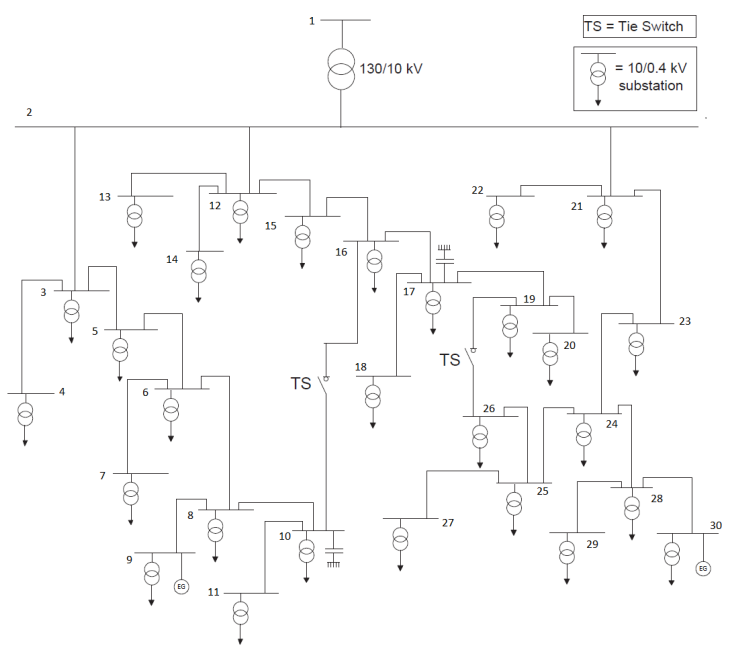

Figure 2. Test system: 30-Bus Gothenburg distribution Network [17].

TABLE I. PARAMETERS OF TRANSFORMER, EMBEDDED GENERATORS AND CAPACITORS OF GOTHENBURG TEST SYSTEM.

\begin{tabular}{|l|l|l|}
\hline & Size & Info \\
\hline & $25 \mathrm{MVA}$ & \\
T1 & $130 / 10 \mathrm{kV}$ & LTC, 9 tap positions, each tap step 5\% \\
\hline EG1 & $200 \mathrm{~kW}$ & $\pm 150 \mathrm{kvar}, 7$ fixed level (steps of 50 kvar) \\
\hline EG2 & $300 \mathrm{~kW}$ & $\pm 150 \mathrm{kvar}, 7$ fixed level (steps of 50 kvar) \\
\hline C1 & $300 \mathrm{kVar}$ & 3 fixed capacitor bank, each 100 kvar \\
\hline C2 & $360 \mathrm{kVar}$ & 3 fixed capacitor bank, each 120 kvar \\
\hline
\end{tabular}

TABLE II. RESULT AND STATISTICS FROM STOCHASTIC VVO

\begin{tabular}{|c|c|c|c|}
\hline $\begin{array}{c}\text { Expected } \\
\text { System Total } \\
\text { Loss } \\
\end{array}$ & $\begin{array}{c}\text { Computation } \\
\text { Time } \\
\text { (20 scenarios) } \\
\end{array}$ & \multicolumn{2}{|c|}{ Optimization Statistics } \\
\hline 7,01 MWh & $300,81 \mathrm{sec}$ & $\begin{array}{l}\text { SINGLE EQUATIONS } \\
\text { SINGLE VARIABLES } \\
\text { DISCRETE VARIABLES }\end{array}$ & $\begin{array}{r}56,257 \\
29,449 \\
5,400\end{array}$ \\
\hline
\end{tabular}

Results show that probability of tap position $\mathrm{t} 7$ and $\mathrm{t} 8$ are close but slightly less than expected tap position (t9). This indicates how the voltage regulation is sensitive to tap changer setting. Also voltage profile along the feeder is always within the acceptable range when loss is minimized for the period.

In this study, 20 load scenarios are used with a cumulative probability above $85 \%$. This means that there is an $85 \%$ chance that actual load conditions are covered by these load scenarios which is which is adequate for such study. By increasing the number of scenarios that percentage will tend toward $100 \%$ while the calculation time will increase, with possibly not a major improvement in the outcome of study, i.e the expected system loss and expected setting for control equipment. Our understanding is that adding more scenarios will not necessarily add value to the results of this stochastic VVO study.

The total computation time in GAMS for stochastic VVO is still in the order of minutes which provides the possibility to use it efficiently for analysis of larger networks and also to examine larger numbers of load scenarios if needed.

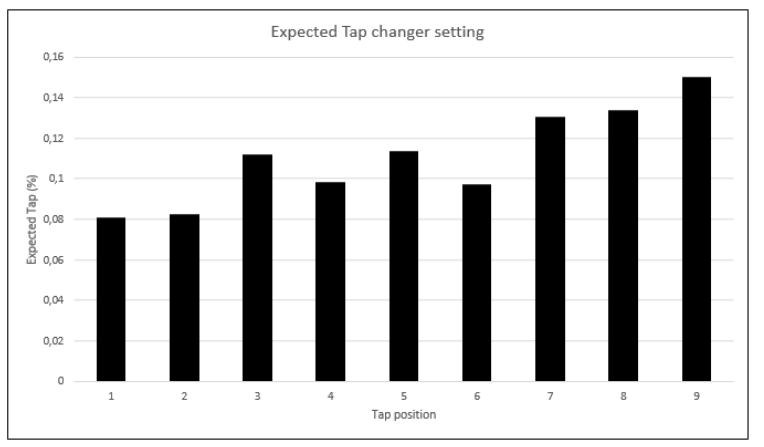

Figure 3. Result from stochastic VVO (expected tap position)

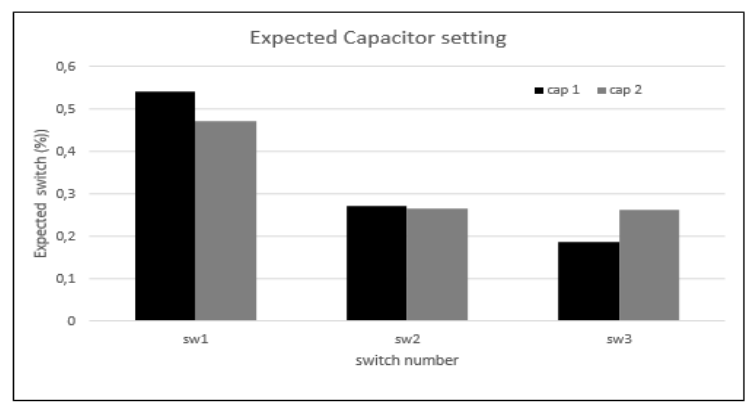

Figure 4. Result from stochastic VVO (expected capacitor switching status)

\section{CONCLUSION}

Results from the stochastic VVO optimization provide valuable information for planning the operation of the distribution network. Based on the method proposed in this paper, the distribution system (DS) planner can start with historical load data and produce any arbitrary number of load scenarios. These load scenarios can be fed into a VVO engine and the outcome is valuable information related to the most probable or the expected operating condition of the network based on those load forecasts. Of course the accuracy of load forecasts is always of concern due to uncertainty in actual consumption in the network. 


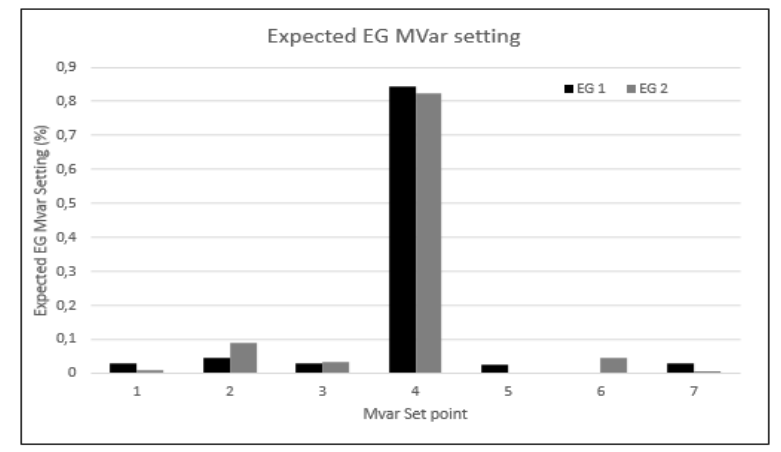

Figure 5. Result from stochastic VVO (expected Mvar level of EGs)
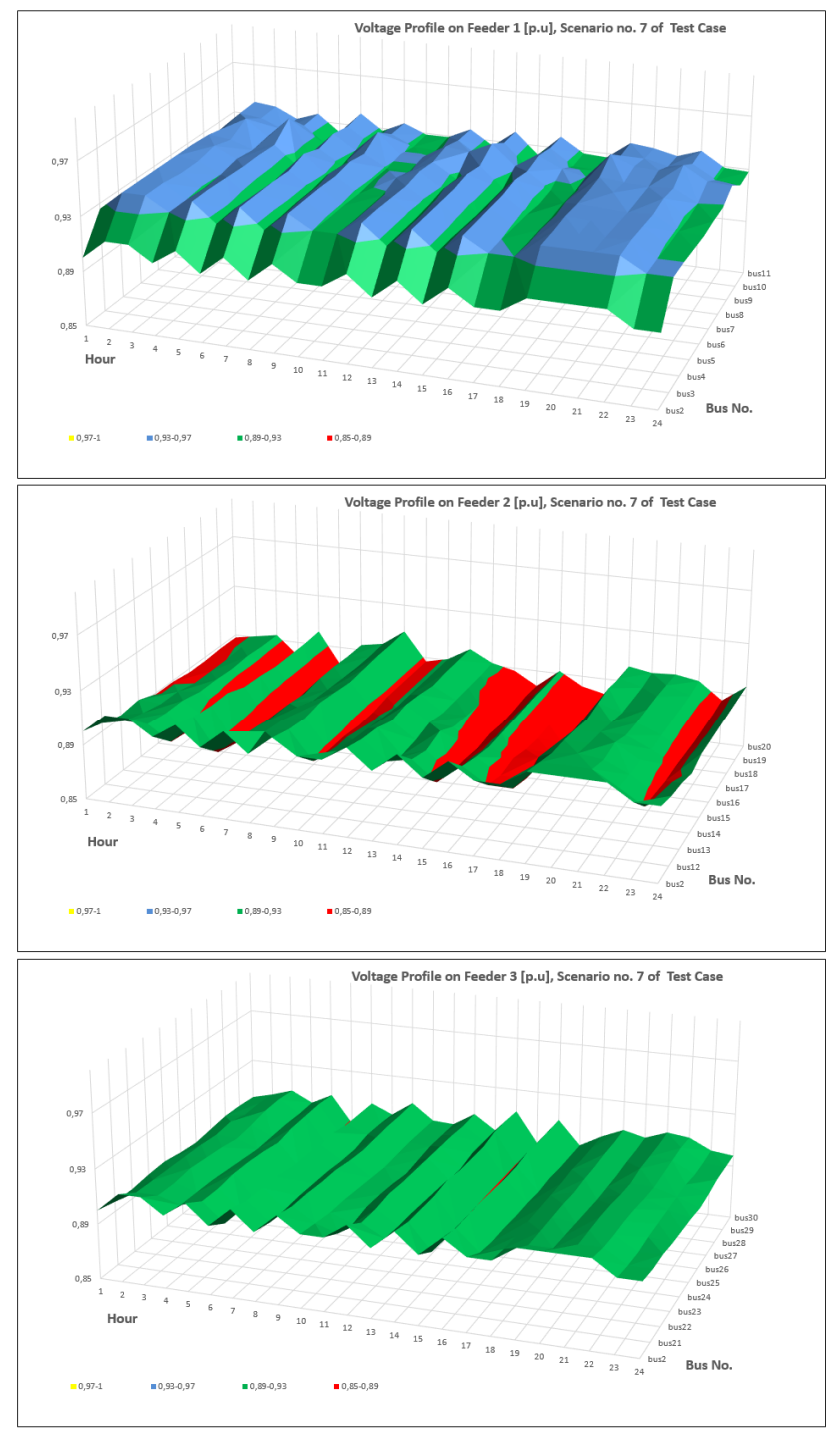

Figure 6. Voltage profile from optimized VVO solution for buses on three feeders ( 24 hour results from $7^{\text {th }}$ scenario)

In this paper the results from implementation of a stochastic Volt/VAR optimization problem for a planning use have been presented. By using the historical load data processed by ARMA model, a limited number of forecasted load scenarios are fed to an MILP VVO engine to give expected system loss and most probable optimal control setting. Based on the quality of results and the short computation time, we expect that applying this method for larger size test systems is practical and promising. It is noted that the discussed model does not include objective elements such as cost of dispatching embedded generations or cost functions related to switching capacitor or tap changer control. Those objectives which have different characteristics can be added to this VVO model as continuation of this work.

\section{REFERENCES}

[1] R. Uluski, "VVC in the Smart Grid era," IEEE Power and Energy Society General Meeting, Minneapolis, MN, July 2010, pp. 1-7.

[2] I. Roytelman and B. Wee, "Volt/Var control algorithm for modern distribution management system," IEEE Transactions on Power Systems, vol. 10, no. 3, pp. 1454-1460, 1995.

[3] Smart Distribution Systems for a Low Carbon Energy Future Workshop, CIRED workshop, 6 June 2011, Frankfurt, Germany.

[4] R. Baldick, F.F. Wu, Efficient integer optimization algorithms for optimal coordination of capacitors and regulators, IEEE Transactions on Power Systems 5 (1990) 805-812.

[5] A. Augugliaro, L. Dusonchet, S. Favuzza, E. Riva Sanseverino, Voltage regulation and power losses minimization in automated distribution networks by an evolutionary multi objective approach, IEEE Transactions on Power Systems 19 (2004) 1516-1527.

[6] M.B. Liu, C.A. Canizares, W. Huang, Reactive power and voltage control in distribution systems with limited switching operations, IEEE Transactions on Power Systems 24 (2009) 889-899.

[7] X. Feng, W. Peterson, and F. Yang, "Implementation of control center based voltage and var optimization in distribution management system," IEEE PES Transmission and Distribution Conference and Exposition, 2010, pp. 1-6, 2010.

[8] S. Rahimi, M. Marinelli, F. Silvestro, "Evaluation of requirements for Volt/Var Control and Optimization function in Distribution Management Systems", 2nd IEEE- International Energy Conference and Exhibition (EnergyCon2012), Firenze, 9 - 12 September 2012,

[9] S. Rahimi, "Advanced DMS Applications for Operation and Control of Distribution Networks in the framework of Smart Grids: a Volt/VAR Optimization Function," Ph.D. Thesis, University of Genova, Feb 2015.

[10] S. Rahimi, Y. Tohidi, S. Massucco, F. Silvestro, M.R.Hesamzadeh, "Applying Full MILP Model to Volt-Var Optimization Problem for MV Distribution Networks", ISGT 2014, Istanbul, Oct 2014.

[11] A. Borghetti, Using mixed integer programming for the volt/var optimization in distribution feeders, Electric Power Systems Research, Volume 98, May 2013, Pages 39-50

[12] R. E. Rosenthal, "GAMS, a user's guide," GAMS Development Corporation, Washington DC, USA, July 2013.

[13] Y. Liu, P. Zhang, X. Qiu, Optimal volt/var control in distribution systems, International journal of Electrical Power and Energy Systems 24 (2002) 271-276.

[14] Brockwell, P.J. \& Davis, R.A. Introduction to Time Series and Forecasting. Springer Texts in Statistics. Second Edition. New York: Springer-Verlag. 2002.

[15] Elisabeth D. Root, Introduction to Quantitative Methods, Course material, GEOG 4023/5023, Spring 2011, available online: http://www.colorado.edu/geography/class_homepages/geog_4023_s11/ lectures.html accessed 2014-10-08

[16] G. E. P. Box, G. M. Jenkins and G. C. Reinsel, Time Series Analysis: Forecasting and Control. 3rd edition. New Jersey: Prentice-Hall, 1994.

[17] David Steen, Tuan Le, Ola Carlson and Lina Bertling, "Assessment of Electric Vehicle Charging Scenarios Based on Demographical Data." IEEE Trans. on Smart Grid Technology, VOL. 3, NO. 3, Sep 2012.

[18] H. Akaike, "Factor analysis and AIC," Psychometrika, vol. 52, 1987.

[19] K. Burnham and D. Anderson, Model selection and multi-model inference: a practical information-theoretic approach. Springer Verlag, 2002. 